\title{
Dual Somatostatin Receptor/FDG PET/CT Imaging in Metastatic Neuroendocrine Tumours: Proposal for a Novel Grading Scheme with Prognostic Significance
}

\author{
David LH Chan, Nick Pavlakis, Geoffrey P Schembri, Elizabeth J Bernard, Edward Hsiao, Aimee Hayes, \\ Tristan Barnes, Connie Diakos, Mustafa Khasraw, Jaswinder Samra, Enid Eslick, Paul J Roach, Alexander \\ Engel, Stephen J Clarke and Dale L Bailey ${ }^{凶}$ \\ Departments of Nuclear Medicine, Medical Oncology and Surgery, Royal North Shore Hospital, Sydney Medical School and Faculty of Health Sciences, \\ University of Sydney, and Sydney Vital Translational Cancer Research Centre, Sydney, Australia \\ $\triangle$ Corresponding author: Dale L Bailey PhD, M: Department of Nuclear Medicine, Royal North Shore Hospital, St Leonards, Australia 2065. E: \\ Dale.Bailey@sydney.edu.au; P: +61 29926 4440; F: +61 299264099 \\ (C) Ivyspring International Publisher. This is an open access article distributed under the terms of the Creative Commons Attribution (CC BY-NC) license \\ (https://creativecommons.org/licenses/by-nc/4.0/). See http://ivyspring.com/terms for full terms and conditions.
}

Received: 2016.10.24; Accepted: 2016.12.17; Published: 2017.03.01

\begin{abstract}
Background: PET scans using FDG and somatostatin receptor imaging agents have both been used to study neuroendocrine tumours. Most reports have documented the sensitivity and specificity of each radiopharmaceutical independently, and even suggested the superiority of one over the other for different grades of disease.

Aim: The aim of this work was to develop a grading scheme that describes the joint results of both the FDG and somatostatin receptor imaging PET scans in staging subjects with neuroendocrine tumours in a single combined parameter. The grading scheme that has been developed is referred to as the NETPET grade.

Methods: This is a retrospective study which assessed subjects who had both FDG and somatostatin receptor PET imaging at our institution within 31 days of each other. The NETPET grade was assigned by experienced nuclear medicine physicians and compared with other clinical data such as WHO grade and overall survival.

Results: In the period 2011-2015 we were able to recruit 62 subjects with histologically proven metastatic neuroendocrine tumour for review. The NETPET grade incorporating both the FDG and somatostatin receptor imaging results was significantly correlated with overall survival by univariate analysis $(p=0.0018)$, whereas in this cohort the WHO grade at the time of diagnosis did not correlate with survival.

Conclusions: The NETPET grade has promise as a prognostic imaging biomarker in neuroendocrine tumours. It permits the capturing of the complexity of dual radiotracer imaging in a single parameter which describes the subjects' disease and is readily amenable to use in patient management and further research.
\end{abstract}

Key words: PET scans, Neuroendocrine tumour

\section{Introduction}

Neuroendocrine tumour (NET) is an umbrella term for a group of heterogeneous cancers which develop from secretory cells found throughout the body and which are particularly concentrated in the gastrointestinal system, pancreas and lung. These cells are referred to as the diffuse endocrine system to separate them from the discrete endocrine organs such as pituitary, thyroid, parathyroid and adrenal glands. 
The term neuroendocrine implies that these cells receive some neural connections ("neuro") and have the ability to secrete hormones ("endocrine"). Neuroendocrine tumours vary widely in anatomical site, clinical course, management and prognosis. Given that prognosis can range from weeks to many years for metastatic disease, accurate prognostication is vital to guide optimal management of disease. Classically, tumour grade and TNM (Tumour-Node-Metastasis) staging have been employed for this purpose. However, tumour grading is prone to sampling error due to intra- and inter-lesional heterogeneity. TNM stage alone cannot fully characterise the biological aggressiveness of a neuroendocrine tumour.

Nuclear medicine functional imaging has been increasingly employed in recent years in the assessment of NETs to inform prognosis and guide management. Gallium-68 $\left({ }^{68} \mathrm{Ga}\right)$ labelled radiopharmaceuticals such as the DOTA-conjugated peptide $\left(\mathrm{Tyr}^{3}\right)$-octreotate ("DOTATATE"), or other variants such as DOTANOC and DOTATOC, collectively referred to as SomatoSTatin Receptor Imaging (SSTRI), are agonists targeted at the somatostatin receptors expressed by well-differentiated NET cells that have been used in positron emission tomography (PET) imaging of NETs due to the improved spatial resolution \& sensitivity of PET compared to SPECT (single photon emission computed tomography) imaging with [111In]-Octreotide $\left(\right.$ Octreoscan $\left.^{\circledR}\right)$ [1]. The uptake of SSTRIs is higher in well-differentiated NETs compared to poorly-differentiated NETs, and correlates strongly with $\mathrm{SSTR}_{2 \mathrm{~A}}$ receptor expression. Good uptake of an SSTRI has been shown to correlate with improved overall survival [2] compared to those with poorer SSTRI uptake as measured by $\mathrm{SUV}_{\max }$.

Fluorine-18 $\left.\quad{ }^{18} \mathrm{~F}\right)$ deoxyglucose (FDG) PET avidity, on the other hand, reflects increased tumour metabolic activity. Its prognostic utility for NETs has recently been demonstrated in a prospective study of 98 subjects which showed that tumours demonstrating a higher maximum standardised uptake value (SUV $\left.V_{\max }\right)$ for FDG PET were associated with significantly poorer overall survival [3]. This study also showed that FDG PET was more likely to be positive with increasing histological grade, from $40 \%$ in subjects with WHO Grade 1 disease to $93 \%$ in Grade 3 disease. FDG positivity has been further validated as a predictor of poorer overall survival in a series of 38 subjects with long-term follow-up [4].

The integration of the imaging information from separate SSTRI and FDG PET scans has previously been proposed as a promising comprehensive imaging biomarker [5-8]. The combination of avidity on FDG PET and non-avidity on SSTRI may indicate a high-grade NET. Subjects with significant FDG-positive, SSTRI-negative disease are likely to have metabolically active, aggressive disease and therefore poorer prognosis. Conversely, a subject with SSTRI uptake on all known metastatic NET lesions without FDG uptake is likely to have low-grade, metabolically inactive disease, leading to an indolent disease course and better prognosis.

Integrated SSTRI/FDG PET may also be a predictive biomarker in subjects being considered for peptide receptor radionuclide therapy (PRRT) with, for example, Lutetium-177 [177Lu]-DOTATATE ("Lutate"). Given that SSTR-targeted PRRT delivers a therapeutic radionuclide to areas of somatostatin receptor uptake, FDG-avid lesions that have no appreciable uptake on SSTRI would not be targeted effectively by PRRT. In addition, such lesions are likely to be higher grade in nature, given that de-differentiation of the neuroendocrine cell is associated with a loss of somatostatin receptor expression.

However, several technical challenges make dual SSTRI/FDG reporting more complex than conventional, single scan PET interpretation. Spatial correlation of the lesions in dual SSTRI/FDG PET imaging is being recognised as increasingly important, both for understanding disease activity and suitability for PRRT. If a lesion shows both FDG and SSTRI avidity, this may correlate to moderate grade and clinical aggressiveness. On the other hand, if there are, in fact, two separate lesions, one being FDG-avid alone and the other SSTRI-avid alone, this might indicate the presence of separate high-grade and low-grade pathology at the different sites of disease. The need to co-register two PET scans, interpret varying patterns of avidity and contextualise the findings with prior clinical history and imaging makes the final report complex. Further, summaries of relevant findings may be difficult to extract, especially in a purely text-based report, for the referring physician. This suggests that a mechanism for integrating the imaging results from both scans into a single combined parameter may be useful.

A variety of grading schemes already exist for both morphological and functional imaging in oncology. These include the WHO [9], RECIST [10] and RECIST 1.1 [11], and the Choi [12] criteria for assessing the change in size and number of neoplastic lesions using cross-sectional imaging with $\mathrm{CT}$ and further applicability to MRI, the PERCIST criteria [13] and Deauville grading [14] for assessing FDG PET scans and the Krenning score [15] for characterising the uptake of SSTR-positive lesions imaged with SPECT or PET. While these scores treat the imaging dataset as a single modality assessment at a particular 
point in time, the greater value is in their use to assess changes over time, such as disease progression or response to therapy. As the availability of multi-modality imaging continues to expand and new techniques and radiopharmaceuticals are introduced there is an increasing need to integrate the information obtained from different types of scans. This work reported in this paper is motivated by such a desire.

With the increasing recognition of the role of nuclear medicine in NET imaging and therapy, a so-called "theranostic" paradigm, and the need for accurate prognostic tools, we devised a novel scheme for dual SSTRI/FDG grading ("the NETPET grade") to describe the most metabolically active tumour based on FDG avidity relative to SSTRI avidity. The intent was to develop a simple scoring system to convey the integrated information from both scans to referrers, to standardise reporting to allow easier categorical database entry and searching, and to provide a quantitative tool for future research.

\section{Aims}

The primary aim of this work was to develop a grading scheme (the "NETPET" grade) for dual SSTRI/FDG PET reporting in subjects with metastatic NETs that summarises the information provided in both scans by a single parameter. The grading scheme should reflect both the spatial concordance of the lesions, relative uptake of the respective radiopharmaceuticals, and the extent of disease in the two studies. It was anticipated that the scheme might give some indication of the suitability of the individual subject for PRRT. Secondary benefits include that the grading scheme should improve consistency and standardisation of reporting of the two scans between reporters and will greatly assist in documenting the results of the scans with a parameter that should be readily amenable to inclusion in a database for rapid interrogation and research. The grading scheme was applied retrospectively to investigate the scheme's ability to predict overall survival, and assess correlation with histological grade.

\section{Description of the NETPET Grading Scheme}

The NETPET scoring scheme was devised in consultation with a number of nuclear medicine physicians \& medical oncologists all of whom had $>10$ years of speciality experience. The strategy adopted was to identify the single lesion that was the most FDG-avid relative to its SSTRI uptake, as this is likely to represent the most aggressive phenotype of the disease present in the subject, excluding other non-NET causes of the increase in FDG (e.g., second malignancy, infection, inflammatory or granulomatous disease, recent surgery or trauma, etc). Once this lesion was defined it provides the primary categorisation of the subject, and subsequent further secondary classification reflecting the burden of disease is then assessed. The NETPET grade uses a 0-5 categorical scale and was largely based on the characteristics of the single, initial lesion, with a grade of P1 indicating purely SSTRI-avid disease without FDG uptake in any lesions, and P5 indicating the presence of significant FDG-positive/SSTRI-negative disease, thought to be a very poor prognostic marker; a NETPET grade of P0 indicates a normal scan on both SSTRI and FDG (usually seen after early diagnosis and surgical resection). The high level categorisation of the scan findings is shown in figure 1 , where the relative uptake and spatial correspondence between the SSTRI and FDG scans is assessed based on visual

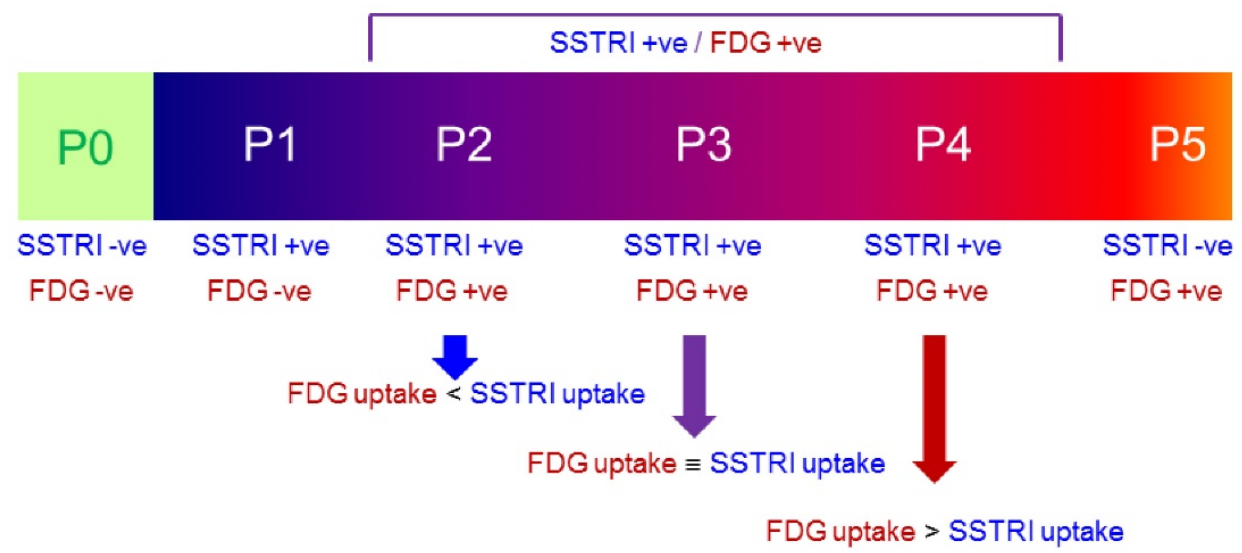

\begin{tabular}{|ll} 
*With image display thresholds set at: & SUV $_{\max }(\mathrm{SSTRI})=15$ \\
& $\mathrm{SUV}_{\max }(\mathrm{FDG})=7$
\end{tabular}

Figure 1. The spectrum of results seen with SSRTI and FDG PET scanning in NETs, split into the categories that provided the basis for the NETPET grading scheme. 
comparison. Below these broad classifiers, we then further characterise the burden of disease based on the number of lesions exhibiting the traits seen in figure 1. A description of the sub-classification is shown in table 1 and a flowchart for the grading scheme with example scans is shown in figure 2.

\section{Methods}

\section{Subject Cohort}

Subjects with metastatic NET who underwent SSTRI (specifically [ $\left.\left.{ }^{68} \mathrm{Ga}\right]-D O T A T A T E\right)$ PET/CT scanning between 2011 and 2015 at Royal North Shore
Hospital, Sydney, a tertiary referral centre for neuroendocrine tumours, were identified. Subjects were included in the study if they had histological confirmation of metastatic NET and underwent [ ${ }^{68} \mathrm{Ga}$ ]-DOTATATE and FDG PET imaging within 31 days of each other. In the case of multiple pairs of eligible scans in the same individual, the earliest pair acquired after histological diagnosis of disease was chosen. This study was approved by the Northern Sydney Local Health District Human Research Ethics Committee (LNR/16/HAWKE/10).

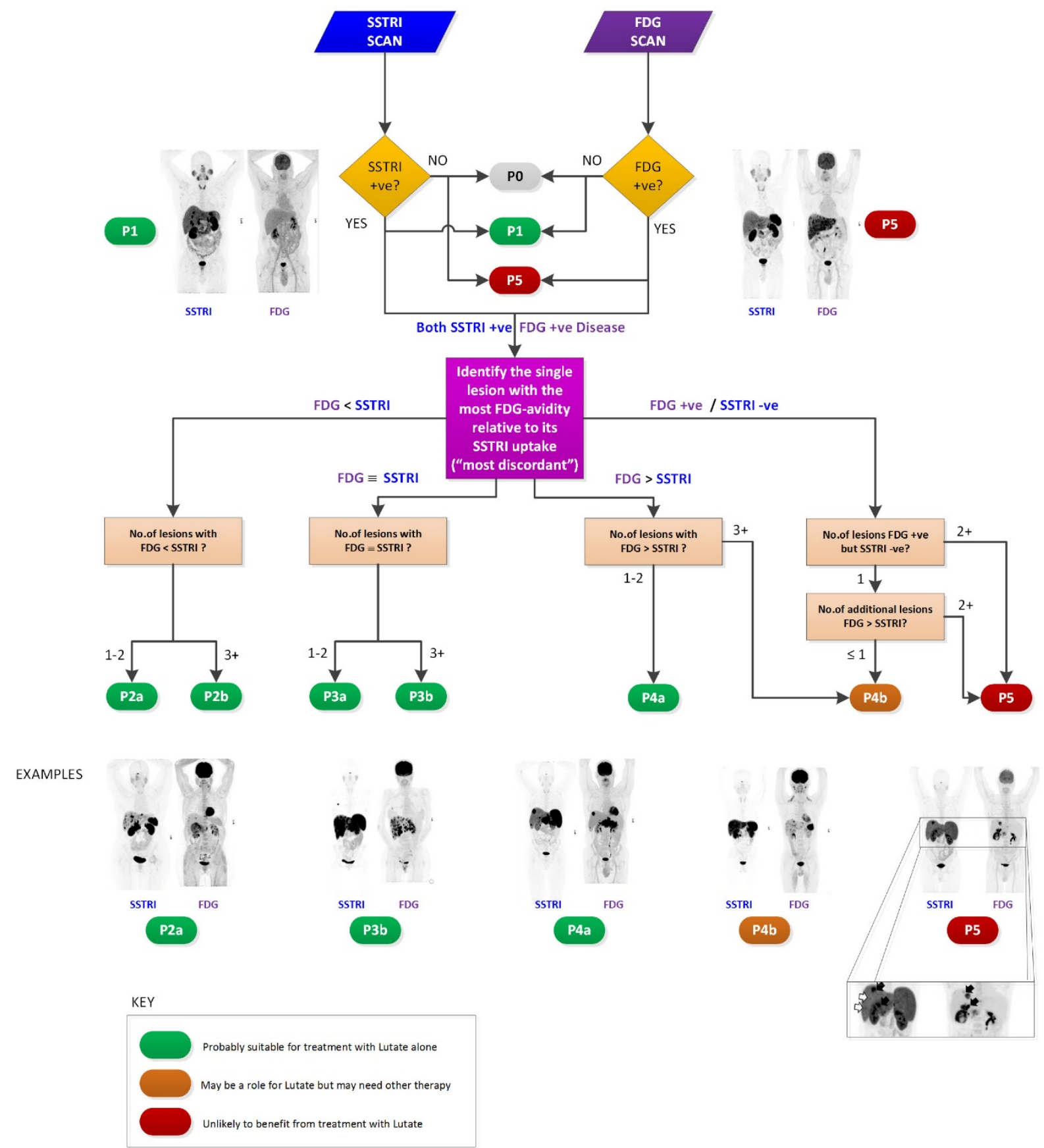

Figure 2. NETPET grading flowchart with selected example images shown. 
Table 1. The categories of NETPET grading descriptors (the colour scheme corresponds to the colours assigned in the flowchart) with green indicating that PRRT may be a potential therapy, amber indicating that PRRT may or may not be useful as a mono-therapy, and red indicating that PRRT alone is unlikely to be an effective therapy).

\begin{tabular}{|c|c|c|c|c|}
\hline $\begin{array}{c}\text { NETPET } \\
\text { Grade }\end{array}$ & $\begin{array}{l}\text { SSTRI } \\
\text { scan } \\
\text { status }\end{array}$ & $\begin{array}{l}\text { FDG } \\
\text { scan } \\
\text { status }\end{array}$ & $\begin{array}{l}\text { Description of target lesion - the single } \\
\text { lesion most FDG avid relative to SSTRI } \\
\text { (no. of lesions with this characteristic) }\end{array}$ & Secondary Characteristic \\
\hline PO & - & - & N/A & - \\
\hline P1 & + & - & N/A & - \\
\hline $\mathrm{P} 2 \mathrm{a}$ & + & + & FDG less avid than SSTRI ( $1-2$ lesions) & - \\
\hline $\mathrm{P} 2 \mathrm{~b}$ & + & + & FDG less avid than SSTRI (3+ lesions) & - \\
\hline P3a & + & + & FDG equivalent to SSTRI ( $1-2$ lesions) & - \\
\hline P3b & + & + & FDG equivalent to SSTRI ( $3+$ lesions) & - \\
\hline P4a & + & + & FDG greater than SSTRI (1-2 lesions) & - \\
\hline $\mathrm{P} 4 \mathrm{~b}$ & + & + & FDG greater than SSTRI (3+ lesions) & - \\
\hline $\mathrm{P} 4 \mathrm{~b}$ & + & + & FDG +ve / SSTRI -ve (1 lesion) & With 1 additional lesion FDG > SSTRI \\
\hline P5 & + & + & FDG +ve / SSTRI -ve (1 lesion) & With $2+$ additional lesions FDG > SSTRI \\
\hline P5 & + & + & FDG +ve / SSTRI -ve (2+ lesions) & \\
\hline P5 & - & + & N/A & \\
\hline
\end{tabular}

The primary endpoint of this study was overall survival measured in days from the latter of the two paired PET scans to the date of death or last follow-up. Overall survival was calculated using the Kaplan-Meier method according to NETPET grade, with three grouped cohorts (grade P1, grades P2-P4, grade P5). These cohorts were chosen as P2-4 represents predominant SSTRI+ve/FDG+ve disease, P1 represents SSTRI+ve/FDG-ve disease, and P5 represents significant SSTRI-ve/FDG+ve disease, and finalized after scan interpretation but before any data analysis. The different cohorts were compared using the log-rank test. Univariate and multivariate regressions were performed using the Cox proportional hazards model with the following variables: age, presence/absence of distant extrahepatic disease, histological grade (using the WHO 2010 criteria) and NETPET grade.

\section{Imaging}

All image data were acquired on a state-of-the-art PET/CT with Time-of-Flight (ToF) capabilities and extended axial field of view (Biograph mCT.S/64 PET/CT, Siemens Healthcare, Hoffman Estates, USA). Data were typically acquired as whole body scans (top of skull to mid-thigh), usually requiring 6-8 bed positions in step-and-shoot mode. For the SSTRI scans the subjects were injected with $120-180 \mathrm{MBq}$ of $\left.{ }^{68} \mathrm{Ga}\right]-$ DOTA- $\left(\mathrm{Tyr}^{3}\right)$-octreotate produced in-house [16] with imaging commencing approximately 50 mins after injection with whole body low-dose CT followed by the PET acquisition of $180 \mathrm{secs} / \mathrm{bed}$. The subjects were advised to cease all somatostatin analogues four weeks prior to the scan. For the $\left.{ }^{18} \mathrm{~F}\right]-F D G$ scans, subjects were required to fast for at least 6 hours prior to the scan and blood glucose levels were checked to ensure they were in the range 4-11 mmol/L. Subjects were administered a standard amount of $250 \mathrm{MBq}$ of FDG if their weight was $\leq 90 \mathrm{~kg}$ or $300 \mathrm{MBq}$ if $>90 \mathrm{~kg}$. At approximately 50 mins after injection of the FDG scanning commenced with whole body low-dose CT followed by the PET acquisition of 150 secs/bed.

All reconstructions were performed using 3D OSEM with 2 iterations and 21 subsets, applied in conjunction with a matched Gaussian post-reconstruction filter of $5 \mathrm{~mm}$ FWHM. Reconstruction included a depth-dependent spatial resolution recovery algorithm ("TrueX", Siemens Healthcare, Hoffman Estates, USA) in addition to standard corrections for random coincidences, scattered photons and attenuation. An example pair of scans from a single subject is shown in figure 3 .

Each pair of scans was read independently by two out of three experienced nuclear medicine physicians (GS, EH, EB) as available, with all pertinent clinical information at the time of scanning provided but without knowledge of subsequent scan findings or clinical outcomes. For cases where the two physicians disagreed, the scans were reviewed together to reach a consensus. If consensus could not be reached, the third physician was asked to arbitrate and decide the final grade.

\section{Criteria for Reporting FDG and SSTRI Positivity}

Scans were graded subjectively by visual interpretation on a dedicated nuclear medicine reporting workstation (Syngo.via, Siemens Healthcare) with both scans displayed simultaneously in transverse, coronal and sagittal planes accompanied by a rotating MIP ciné of the PET data, 
with the two image sets anatomically co-registered and locked so that all moved in synchrony. The scans were initially windowed with preset values for SUV

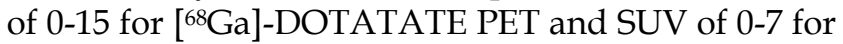
FDG PET, as these were the values used for reporting in clinical practice (appreciating the greater tumour-to-background ratio in general with

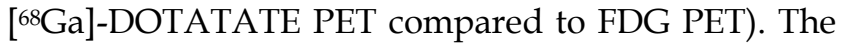
readers had full access to all software tools (window/level adjustment, alternative colour tables, SUVs within a region of interest (ROI), distance-defining callipers, etc). Positivity on an FDG scan was defined as uptake which was greater than that regarded as physiological for the tissue in question (liver, lesion, lymph node, etc). For the SSTRI PET, any lesion with intensity greater than expected that was not a known area with physiologic normally increased uptake (e.g., pituitary gland, uncinate process of pancreas, kidney, spleen) was considered positive.

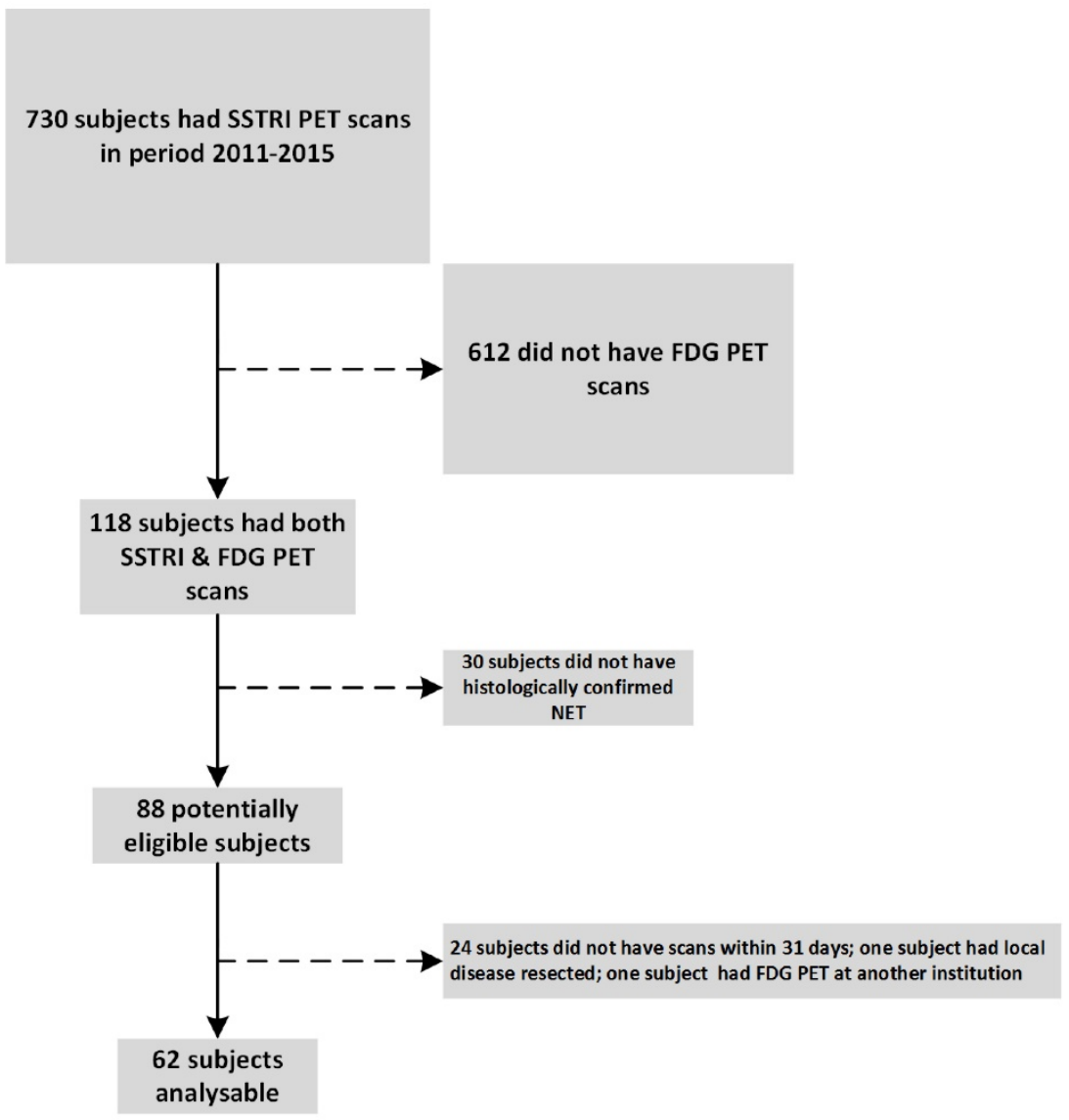

Figure 4. Trial cohort selection

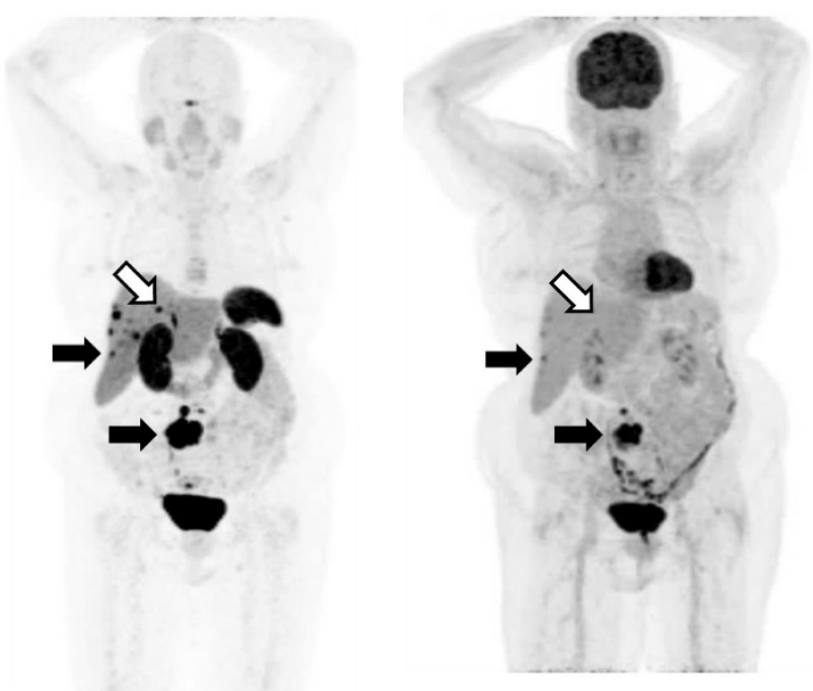

Figure 3. An example of an SSTRI ([68Ga]-DOTATATE) and FDG scan pair. The MIP (maximum intensity projections) images from the respective PET data sets are shown. The subject has SSTRI-dominant disease which exhibits some lesions with spatially concordant FDG uptake (closed arrows). While there are some sites of disease which are SSTRI-positive but FDG-negative (open arrows), importantly, there are no sites which are FDG-positive and SSTRI-negative. The example shown demonstrates a NETPET score of P3a, indicating that (i) the scans are positive on both SSTRI and FDG, and (ii) there were up to 2 lesions demonstrating approximately equivalent uptake between SSTRI and FDG, with the remaining lesions demonstrating greater SSTRI avidity than FDG.

\section{Statistical analysis}

Correlation

between histological grade and NETPET grade was assessed using Spearman's correlation coefficient. Calculations were carried out using SAS (version 9.4) and Graphpad Prism 7 (GraphPad Software Inc., California USA) and R [17]. In these analyses the level for significance was set at $p=0.05$ or less. With regard to overall survival, Kaplan-Meier curves were constructed and compared using the log-rank test [18]. Univariate and multivariate analyses were performed based on the NETPET grade and the recognised prognostic factors of age, WHO 2010 Grade and presence of distant extrahepatic disease using the Cox proportional-hazards model.

\section{Results}

In the period 2011 - 2015 we performed $730 \quad\left[{ }^{68} \mathrm{Ga}\right]$-DOTATATE PET scans. Of these, 118 subjects had both FDG and [ $\left.{ }^{68} \mathrm{Ga}\right]$-DOTATATE PET of which 62 subjects were 
identified who met the inclusion criteria including histologically confirmed metastatic NET and who had the two PET scans within 31 days of each other (Fig.4). One patient underwent FDG PET shortly prior to resection of one liver metastasis as the only site of disease. As the focus of the current manuscript was on the correlation between dual PET scan and the grade of remaining tumour in the patient, this patient was excluded.

The subject characteristics of this cohort are summarised in Table 2. The subjects ranged in age from $27-82$ years (median $=59.5 \mathrm{yrs}$ ). The median follow up for patients still alive was 27 months (range 11.5 - 61.3 months).

The distribution of primary tumour sites is detailed in table 2.

When the NETPET grading system was applied to the above subjects' scans, 11 subjects were graded as P1, 33 as P2-4 and 18 as P5. The median overall survival was not reached for subjects graded P1 or P2-4. For subjects graded P5 the median overall survival was 11 months ( $\mathrm{p}=0.0018$ by log rank test, Fig.5).

On univariate analyses, overall survival was significantly associated with NETPET grade $(p=0.0018)$ but not age $(p=0.19)$, the presence of extrahepatic disease $(p=0.11)$ or histological grade $(p=0.29)$. As only NETPET grade was significant on univariate analysis, multivariate analysis was not performed.

NETPET grade was significantly associated with WHO 2010 histological grade (Spearman's test for correlation, $\mathrm{r}=0.57, \mathrm{p}<0.00001)$.

Table 2. Baseline subject characteristics

\begin{tabular}{ll}
\hline Characteristic & Number of subjects (\%) \\
\hline Gender & \\
Female & $24(39 \%)$ \\
Male & $38(61 \%)$ \\
WHO 2010 Histological Grade & \\
Grade 1 & \\
Grade 2 & $14(23 \%)$ \\
Grade 3 & $33(53 \%)$ \\
Unknown & $12(19 \%)$ \\
& $3(5 \%)$ \\
Site of primary & \\
Pancreas & \\
Midgut & \\
Other GI & $24(39 \%)$ \\
Lung & $20(32 \%)$ \\
Unknown & $5(8 \%)$ \\
Thymus & $5(8 \%)$ \\
Breast & $3(5 \%)$ \\
& $3(5 \%)$ \\
TOTAL & $2(3 \%)$ \\
\hline
\end{tabular}

Sensitivity analyses were performed investigating the effect of restricting analysis to subjects with GEPNETs. NETPET grade remained a significant predictor of overall survival on both univariate and multivariate analysis (Table 4).

Table 3. Retrospective classification of included patients by NETPET grade and the number treated with Lutate

\begin{tabular}{lll}
\hline Grade & Number & Lutate Treatment? \\
\hline P1 & 11 & 2 \\
P2a & 4 & 3 \\
P2b & 15 & 7 \\
P3a & 1 & 1 \\
P3b & 1 & 0 \\
P4a & 5 & 1 \\
P4b & 7 & 5 \\
P5 & 18 & 2 \\
Total & $\mathbf{6 2}$ & $\mathbf{2 1}$ \\
\hline
\end{tabular}

Table 4. Results of univariate and multivariate analysis for overall survival in patients with GEPNETs

\begin{tabular}{lll}
\hline & Univariate analysis & Multivariate analysis \\
\hline Tumour grade & $\mathrm{p}=0.2117$ & $\mathrm{~N} / \mathrm{A}$ \\
Extrahepatic disease & $\mathrm{p}=0.0297$ & $\mathrm{p}=0.2816$ \\
NETPET grade & $\mathrm{p}<0.0001$ & $\mathrm{p}=0.0009$ \\
Age & $\mathrm{p}=0.4100$ & $\mathrm{~N} / \mathrm{A}$
\end{tabular}

N/A: Analysis not performed as univariate analysis not significant

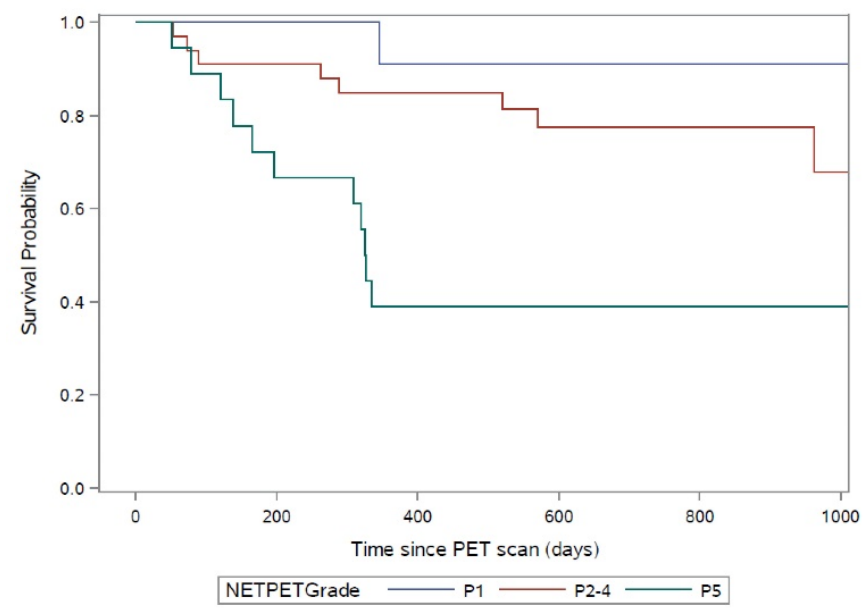

Figure 5. Kaplan-Meier curves for the NETPET subjects grouped as $\mathrm{PI}(\mathrm{N}=11)$, P2-P4 $(\mathrm{N}=33)$ and P5 $(\mathrm{N}=18)$.

\section{Discussion}

Our results have demonstrated that the NETPET scoring system may have prognostic value in subjects with GEPNETs. In our relatively small cohort, histological grade, age and the presence of extrahepatic disease - all suggested prognosticators in NETs - were not significantly associated with outcome on univariate/multivariate analyses, 
whereas the imaging biomarker of NETPET grade remained significant.

To our knowledge, this is the first paper to investigate the prognostic value of an integrated parameter derived from dual SSTRI and FDG PET. A number of previous studies have described the prognostic value of FDG PET in NETs $[3,4,8,19]$ and one has also investigated the utility of Octreoscan in the same study but did not attempt to spatially correlate this with FDG. It is generally accepted that $\left[{ }^{68} \mathrm{Ga}\right]-S S T R I$ PET is superior to Octreoscan SPECT in terms of spatial resolution, detectability, sensitivity and diagnosis in NETs; this may be the reason why dual grading was not attempted in the above paper. One study has also suggested that high SUV on SSTRI PET predicts better outcome [2]. The current paper combines the above two concepts into one classification scheme by creating an intermediate group of subjects with lesions which exhibit uptake on both FDG and SSTRI PET scans (Fig 6).

The above findings also support a potential role for molecular imaging in addition to biopsy to determine tumour aggressiveness in a particular subject (Fig.7). We note that NETPET grade was significantly associated with histological grade - but that it was more predictive of outcome. Histological grade is an inaccurate measure on a subject level because of intra-subject heterogeneity and the evolution of the disease over time, whereas FDG/SSTRI PET may identify the highest-grade, most aggressive lesion by FDG positivity and SSTRI negativity. This may lead to better selection of biopsy site at diagnosis to identify the highest-grade disease. It may also suggest potential changes in tumour behaviour in explaining treatment resistance. In time, molecular imaging may even make regrading by histological biopsy obsolete, especially in the context of PRRT as the treatment modality.

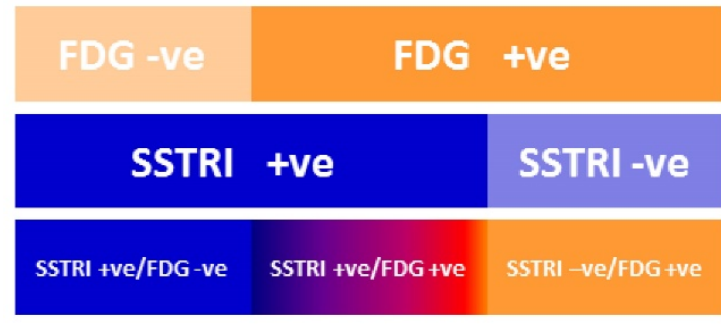

\section{FDG ASSESSMENT}

SSTRI ASSESSMENT

\section{NETPET ASSESSMENT}

Figure 6. Graphical representation of the concept behind the classification scheme

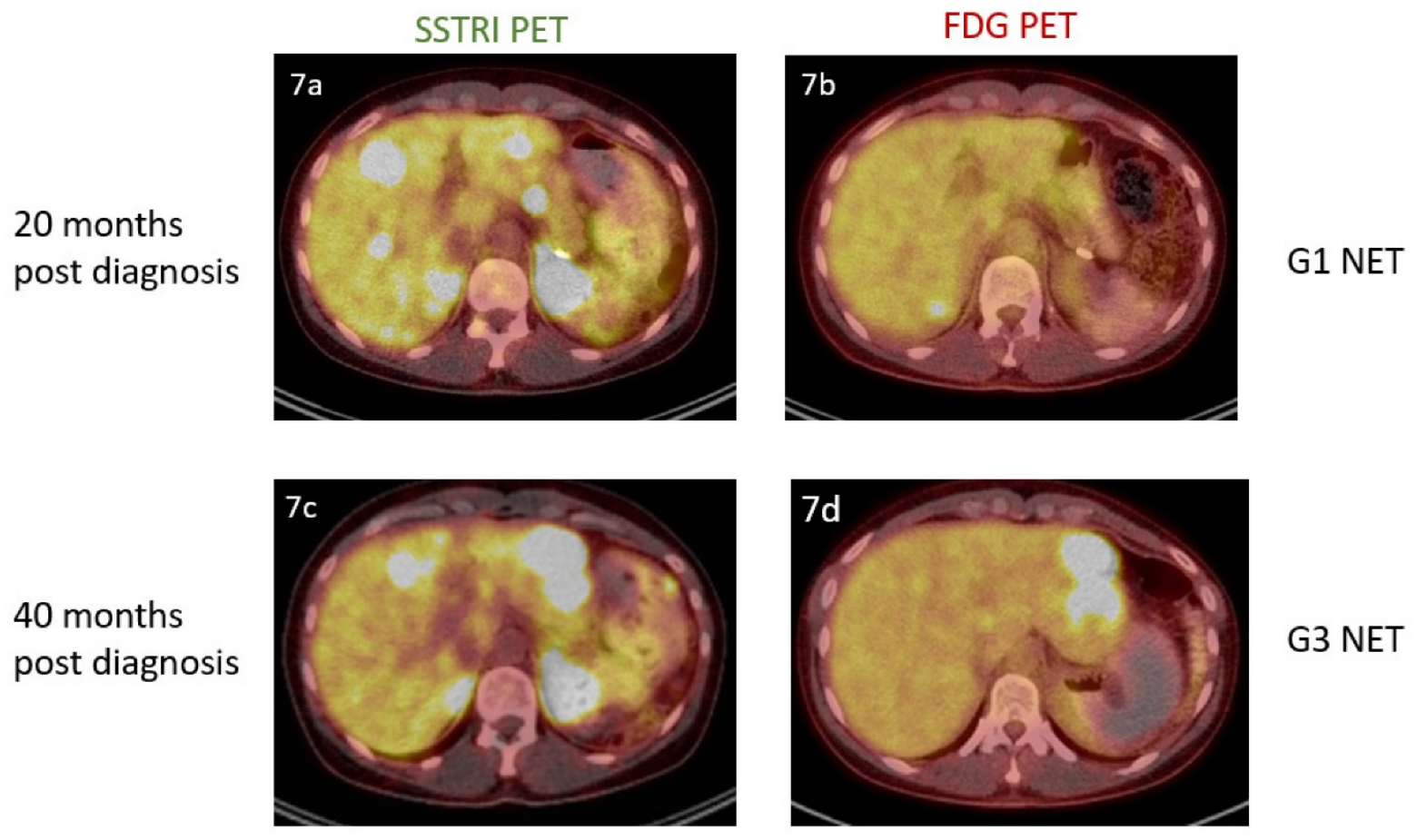

Figure 7. A 36 year old female was incidentally found to have obstructive liver function in a workup for an unrelated procedure. Computed tomography of the abdomen demonstrated a $20 \mathrm{~mm}$ arterially enhancing mass in the tail of the pancreas and multiple enhancing bilobar hepatic lesions. Liver biopsy and subsequent pancreatectomy confirmed the presence of a grade 1 pancreatic neuroendocrine tumour (mitotic count $<1$, Ki-67 index $=1 \%$ ). Dual imaging at this point $(7 \mathrm{a}, 7 \mathrm{~b}$ ) revealed the presence of SSTRI+ve/FDG-ve disease. Repeat imaging 20 months later (7c, 7d) demonstrated progressive disease and the new presence of FDG avidity. She underwent left hepatectomy for this lesion, with histology confirming the presence of a Grade 3 neuroendocrine tumour (mitotic count 20 , Ki-67 index $=30 \%$ ). 
It is somewhat surprising that histological grade was not associated with overall survival given its recognised prognostic impact [20]. However, it must be noted that this is a relatively small and heterogeneous cohort, in comparison to validation studies with larger numbers of subjects with the same site of primary. In our view, this lack of significance merely highlights the prognostic power of the PET scans and the NETPET grading system driven, in part, by the fact that the PET scans provide a total body evaluation.

There are, however, limitations to the current study. The retrospective nature of this small study means that the findings, although intriguing, need to be validated prospectively. The two PET scans were generally not performed on the same day, meaning that shift in location/size of lesions and rapid progression may impede accurate comparison between the scans; this possibility was reduced by restricting analysis to subjects with PET scans within 31 days of each other - a relatively short period for indolent neuroendocrine tumours. The development of a novel grading system also needs to be tested in other centres to establish practical utility and measure inter-rater reliability. A larger cohort may have confirmed the prognostic significance of known determinants of outcome such as histological grade.

Although it may be too early at present to adopt NETPET scoring in routine clinical practice, the current study does suggest multiple avenues for further research. Apart from confirmatory prospective studies, the utility of dual PET imaging in changing management decisions should be investigated given the considerable cost of imaging, and also of therapy. There is increasing interest in the use of SSTRI PET as a predictive biomarker for PRRT, given its reliance on NET cells expressing somatostatin receptors in order for the therapeutic radionuclide to be taken up and internalised by the NET cell. SSTRI PET SUV max $_{\text {may }}$ predict response to PRRT, although FDG-positive/SSTRI-negative lesions may also respond to PRRT [21-23]. Our scheme suggests that subjects with NETPET grade of P4b and P5 - that is, subjects with significant FDG-positive/SSTRInegative disease - may not derive adequate tumour control from PRRT alone and should have systemic chemotherapy instead, and that this hypothesis should be investigated in future studies.

\section{Conclusions}

Dual FDG/SSTRI PET imaging divides subjects into three subgroups - solely SSTRI-positive, SSTRI-positive/FDG-positive disease, and SSTRI-negative/FDG-positive (multiple lesions that exhibit significant FDG/SSTRI discordance). This small retrospective study shows that the above subgroups show significant correlation with overall survival. The utility of dual PET imaging as a prognostic biomarker, as well as the clinical utility of the proposed grading schema, should be investigated in prospective studies. If confirmed, this would provide evidence to support the use of dual PET imaging for a more accurate assessment of subjects with metastatic neuroendocrine tumours.

\section{Acknowledgements}

This work was supported by funding from the Cancer Institute of New South Wales (CINSW) through the Translational Cancer Research Centre programme, of which Sydney Vital is a recipient.

David Chan received support from Sydney Vital for this work.

Aimee Hayes currently receives support from Sydney Vital.

Dale Bailey receives on-going support for the Neuro-Endocrine Tumour programme from Sydney Vital.

\section{Competing Interests}

The authors have declared that no competing interest exists.

\section{References}

1. Mojtahedi A, Thamake S, Tworowska I, Ranganathan D, Delpassand ES. The value of $68 \mathrm{Ga}$-DOTATATE PET/CT in diagnosis and management of neuroendocrine tumors compared to current FDA approved imaging modalities: a review of literature. Am J Nucl Med Mol Imaging. 2014; 4: 426-34.

2. Campana D, Ambrosini V, Pezzilli R, Fanti S, Labate AM, Santini D, et al. Standardized uptake values of 68Ga-DOTANOC PET: a promising prognostic tool in neuroendocrine tumors. J Nucl Med. 2010; 51: 353-9.

3. Binderup T, Knigge U, Loft A, Federspiel B, Kjaer A. 18F-fluorodeoxyglucose positron emission tomography predicts survival of patients with neuroendocrine tumors. Clin Cancer Res. 2010; 16: 978-85.

4. Bahri H, Laurence L, Edeline J, Leghzali H, Devillers A, Raoul JL, et al. High prognostic value of 18F-FDG PET for metastatic gastroenteropancreatic neuroendocrine tumors: a long-term evaluation. J Nucl Med. 2014; 55: 1786-90.

5. Kayani I, Bomanji JB, Groves A, Conway G, Gacinovic S, Win T, et al. Functional imaging of neuroendocrine tumors with combined PET/CT using 68Ga-DOTATATE (DOTA-DPhe1,Tyr3-octreotate) and 18F-FDG. Cancer. 2008; 112: 2447-55.

6. Kayani I, Conry BG, Groves AM, Win T, Dickson J, Caplin M, et al. A comparison of $68 \mathrm{Ga}$-DOTATATE and 18F-FDG PET/CT in pulmonary neuroendocrine tumors. J Nucl Med. 2009; 50: 1927-32.

7. Hofman MS, Hicks RJ. Changing paradigms with molecular imaging of neuroendocrine tumors. Discovery medicine. 2012; 14: 71-81.

8. Nilica B, Waitz D, Stevanovic V, Uprimny C, Kendler D, Buxbaum S, et al. Direct comparison of 68Ga-DOTA-TOC and 18F-FDG PET/CT in the follow-up of patients with neuroendocrine tumour treated with the first full peptide receptor radionuclide therapy cycle. Eur J Nucl Med Mol Imaging. 2016; 43: 1585-92.

9. Miller AB, Hoogstraten B, Staquet M, Winkler A. Reporting results of cancer treatment. Cancer. 1981; 47: 207-14.

10. Therasse P, Arbuck SG, Eisenhauer EA, Wanders J, Kaplan RS, Rubinstein L, et al. New guidelines to evaluate the response to treatment in solid tumors. European Organization for Research and Treatment of Cancer, National Cancer Institute of the United States, National Cancer Institute of Canada. J Natl Cancer Inst. 2000; 92: 205-16.

11. Eisenhauer EA, Therasse P, Bogaerts J, Schwartz LH, Sargent D, Ford R, et al. New response evaluation criteria in solid tumours: revised RECIST guideline (version 1.1). Eur J Cancer. 2009; 45: 228-47.

12. Choi H. Response evaluation of gastrointestinal stromal tumors. Oncologist. 2008; 13 Suppl 2: 4-7. 
13. Wahl RL, Jacene H, Kasamon $\mathrm{Y}$, Lodge MA. From RECIST to PERCIST: Evolving Considerations for PET response criteria in solid tumors. J Nucl Med. 2009; 50 Suppl 1: 122S-50S.

14. Meignan M, Gallamini A, Meignan M, Gallamini A, Haioun C. Report on the First International Workshop on Interim-PET-Scan in Lymphoma. Leukemia \& lymphoma. 2009; 50: 1257-60.

15. Kwekkeboom DJ, Teunissen JJ, Bakker WH, Kooij PP, de Herder WW, Feelders RA, et al. Radiolabeled somatostatin analog [177Lu-DOTA0,Tyr3]octreotate in patients with endocrine gastroenteropancreatic tumors. J Clin Oncol. 2005; 23: 2754-62.

16. Aslani A, Snowdon GM, Bailey DL, Schembri GP, Bailey EA, Roach PJ. Gallium-68 DOTATATE Production with Automated PET Radiopharmaceutical Synthesis System: A Three Year Experience. Asia Oceania J Nucl Med Biol. 2014; 2: 75-86.

17. R-Core-Team. R: A language and environment for statistical computing. Vienna, Austria: R Foundation for Statistical Computing; 2015.

18. Altman DG. Practical Statistics for Medical Research. London: Chapman \& Hall; 1991. p203, 531.

19. Ezziddin S, Adler L, Sabet A, Poppel TD, Grabellus F, Yuce A, et al. Prognostic stratification of metastatic gastroenteropancreatic neuroendocrine neoplasms by 18F-FDG PET: feasibility of a metabolic grading system. J Nucl Med. 2014; 55: $1260-6$.

20. La Rosa S, Inzani F, Vanoli A, Klersy C, Dainese L, Rindi G, et al. Histologic characterization and improved prognostic evaluation of 209 gastric neuroendocrine neoplasms. Hum Pathol. 2011; 42: 1373-84.

21. Kong CB, Byun BH, Lim I, Choi CW, Lim SM, Song WS, et al. 18F-FDG PET SUVmax as an indicator of histopathologic response after neoadjuvant chemotherapy in extremity osteosarcoma. Eur J Nucl Med Mol Imaging. 2013; 40: 728-36

22. Kong G, Thompson M, Collins M, Herschtal A, Hofman MS, Johnston V, et al. Assessment of predictors of response and long-term survival of patients with neuroendocrine tumour treated with peptide receptor chemoradionuclide therapy (PRCRT). Eur J Nucl Med Mol Imaging. 2014; 41: 1831-44.

23. Kratochwil C, Stefanova M, Mavriopoulou E, Holland-Letz T, Dimitrakopoulou-Strauss A, Afshar-Oromieh A, et al. SUV of [68Ga]DOTATOC-PET/CT Predicts Response Probability of PRRT in Neuroendocrine Tumors. Mol Imaging Biol. 2015; 17: 313-8. 